\title{
Management of moderate to severe psoriasis in patients with metabolic comorbidities
}

\author{
Paolo Gisondi $^{1}$, , Arturo Galvan ${ }^{1}$, Luca Idolazzi ${ }^{2}$ and Giampiero Girolomoni ${ }^{1}$ \\ 1 Section of Dermatology and Venereology, Department of Medicine, University of Verona, Verona, Italy \\ ${ }^{2}$ Section of Rheumatology, University of Verona, Verona, Italy
}

\author{
Edited by: \\ Wei-Sheng Chong, National Skin \\ Centre, Singapore \\ Reviewed by: \\ Miriam Wittmann, University of \\ Leeds, UK \\ Hideki Ogura, Osaka University, Japan \\ Fu-Tong Liu, University of California \\ Davis, USA \\ Hidetoshi Takahashi, Asahikawa \\ Medical University, Japan \\ ${ }^{*}$ Correspondence: \\ Paolo Gisondi, Section of \\ Dermatology, Department of \\ Medicine, University of Verona, \\ Piazzale A, Stefani 1, Verona 1-37126, \\ Italy \\ e-mail:paolo.gisondi@univr.it
}

Psoriasis is a chronic inflammatory skin disease affecting 2-3\% of worldwide population. The extent of skin involvement is variable, ranging from a few localized plaques to generalized involvement. Moderate to severe psoriasis ( $>10 \%$ of body surface area) is frequently associated with psoriatic arthritis and metabolic diseases, like abdominal obesity, diabetes, non-alcoholic fatty liver disease, dyslipidemia, metabolic syndrome, and chronic kidney disease. A common genetic background as well as several acquired risk factors links psoriasis to comorbidities. From a clinical prespective, the understanding of the patients in the context of these comorbidities is very important to ensure that treatment is tailored to meet the individual patient needs. Indeed, some pharmacological treatments may negatively affect cardio-metabolic comorbidities, and have important interactions with drugs that are commonly used to treat them. Non-pharmacological intervention such as diet, smoking cessation, and physical exercise could both improve the response to treatments for psoriasis and reduce the cardiovascular risk.

Keywords: psoriasis, comorbidities, conventional therapies, biological therapies

\section{INTRODUCTION}

Psoriasis is an immune-mediated chronic inflammatory disease affecting approximately $2-3 \%$ of Caucasian population (1). It can occur at any age, although the majority of cases develop before the age of 40 years and it is uncommon in children. Psoriasis is a complex disease with a strong genetic background (1). So far, about 20 chromosome regions have been proposed to harbor psoriasis susceptibility genes, which affect mostly innate and adaptive immune responses. The locus carrying the highest risk is the class I region of the major histocompatibility antigen cluster, which harbor the human leukocyte antigen Cw6 and is associated to early onset psoriasis (2). The current understanding of the molecular pathogenesis of psoriasis assigns central importance to the interaction between acquired and innate immunity (3). Chronic plaque psoriasis is the most common type of the disease (4). The extent of skin involvement is widely variable, ranging from a few localized plaques at extensor sites to generalized involvement. Moderate to severe psoriasis is defined if the body surface involvement is $>10 \%$. Patients with psoriasis, like those with other major medical disorders, have a worse quality of life, reduced life expectancy as well as employment and income (5).

\section{CHRONIC PLAQUE PSORIASIS AND METABOLIC COMORBIDITIES}

Several epidemiological studies have confirmed that moderate to severe psoriasis is strongly associated with cardio-metabolic disorders including hypertension, obesity, type 2 diabetes, dyslipidemia, non-alcoholic fatty liver disease (NAFLD), metabolic syndrome, and chronic kidney disease (CKD) (6). In particular, patients with psoriasis are more frequently overweight or obese than the general population, and the severity of psoriasis is correlated to body mass index (BMI) $(7,8)$. The association between obesity and psoriasis has been recently confirmed also in pediatric patients (9). Obesity generally precedes the development of psoriasis and the BMI is correlated to an increased risk of incident psoriasis. Several measures of adiposity, including BMI, waist, and hip circumference and waist-hip ratio have been reported as independent risk factors for the development of psoriasis and psoriatic arthritis (PsA) (10). The relationships between psoriasis and obesity may be largely explained by the complex properties of the adipose tissue. Indeed, the adipose tissue is not only a storage fat organ but an active endocrine organ with many secretory products, such as free fatty acids, adipocyte-derived hormones, and pro-inflammatory adipokines, including chemerin, resistin, visfatin, interleukin (IL)6 , and tumor necrosis factor (TNF)- $\alpha$ (11). In addition, a recent meta-analysis reported that psoriasis is a risk factor for both prevalent and incident type 2 diabetes mellitus $(12,13)$.

Visceral obesity and type 2 diabetes are two main components of the metabolic syndrome. The metabolic syndrome is a set of metabolic disorders, in particular insulin resistance, which may confer a higher pro-inflammatory and pro-thrombotic risk. The diagnosis of metabolic syndrome is confirmed in presence of three or more of the following conditions including abdominal obesity (waist circumference $>102 \mathrm{~cm}$ in men; $>88 \mathrm{~cm}$ in women), elevated serum triglycerides $[>150 \mathrm{mg} / \mathrm{dl}(1.7 \mathrm{mmol} / \mathrm{l})$ or treatment], low HDL cholesterol [men $<40 \mathrm{mg} / \mathrm{dl}(1 \mathrm{mmol} / \mathrm{l})$; women $<50 \mathrm{mg} / \mathrm{dl}(1.3 \mathrm{mmol} / \mathrm{l})$; or treatment], elevated blood pressure $(>130 / 85 \mathrm{mmHg}$ or treatment $)$, and elevated fasting glucose (>100 mg/dL or treatment) (14). In a cross-sectional study, we found that patients with psoriasis had a higher prevalence of metabolic syndrome than those with other inflammatory skin diseases after controlling for sex and age (30.1 vs. 20.6\%, OR: 1.65, 
95\% CI 1.16-2.35) (14). The association between psoriasis and metabolic syndrome has been also recently confirmed in a metaanalysis showing that psoriasis carries a pooled odds ratio for metabolic syndrome of 2.26 (95\% CI 1.70-3.01) (15). NAFLD is a spectrum of progressive liver disease that encompasses simple steatosis, non-alcoholic steato-hepatitis (NASH), fibrosis, and ultimately cirrhosis. NAFLD is recognized as the hepatic expression of the metabolic syndrome, as these conditions have insulin resistance as a common pathophysiological mechanism (16). We found that the prevalence of NAFLD in patients with chronic plaque psoriasis was remarkably greater than that in non-psoriasis controls ( 47 vs. $28 \%$; $p<0.0001$ ) who were matched by age, sex, and BMI (17). In a recent Dutch study, NAFLD was diagnosed in $46.2 \%$ of patients with psoriasis compared with $33.3 \%$ of the controls $(p=0.005)$ (18). Psoriasis was found associated to NAFLD independently of alcohol consumption, smoking status, presence of metabolic syndrome, and serum levels of alanine aminotransferase (adjusted odds ratio 1.7, 95\% CI 1.1-2.6) (18). NAFLD is an emerging risk factor for cardiovascular diseases (CVD) (19). There is conflicting evidence as to whether psoriasis is associated with increased cardiovascular outcomes. Patients with psoriasis show a higher prevalence of other important CVD risk factors, including smoking, sedentary habit and dyslipidemia, making them more susceptible to development of CVD morbidity and mortality, and particularly young patients with the more severe skin disease (20, 21). Some studies reported that patients with severe psoriasis, but not those with mild disease, are at increased risk of developing acute myocardial infarction and stroke, as well as CVD mortality (22). Uncertainty still remains about whether the increased CVD risk observed in psoriatic patients is directly attributable to psoriasis itself or to the effects of multiple co-existing risk factors (23).

Recently, the association between psoriasis and CKD has also been reported (24). A retrospective cohort study showed in that moderate to severe psoriasis is associated with CKD independently of traditional risk factors. In particular, it was found that $5 \%$ of patients with psoriasis had CKD compared to $2 \%$ of controls. No association was shown in patients with mild disease (with a body surface area $<2 \%$ ). The relative risk of CKD was observed being especially increased in younger patients. Nerveless, despite the attenuation of the association with increasing age, the clinical relevance of the absolute risk of CKD attributable to psoriasis was demonstrated increasing with age. Because of these findings closer monitoring for renal insufficiency, such as routine screening for microalbuminuria and serum creatinine should be performed in patients with moderate to severe disease. Additionally, the risk vs. benefit of potentially nephrotoxic drugs such as cyclosporine in patients with moderate to severe psoriasis should be carefully considered.

The association between psoriasis and comorbidities could be explained considering a common genetic background, the systemic effects of chronic inflammation, insulin resistance, and an unhealthy life-style such as heavy smoking/drinking, over-eating habit, and sedentary life, which are common in patients with psoriasis. A meta-analysis of 4 different studies reported that 10 metabolic gene single nucleotide polymorphisms including those related to CARD14 are closely correlated to both psoriasis and metabolic disorders, such as diabetes, dyslipidemia, and hypertension (25). Psoriasis and obesity-related inflammatory status can fuel reciprocally. Indeed, it is likely that cytokines released from either psoriatic keratinocytes or inflammatory cells infiltrating psoriatic skin might induce systemic insulin resistance, thus favoring the development of type 2 diabetes mellitus (11). On the other hand, it is also possible that the co-existing metabolic comorbidities might directly contribute to exacerbate psoriatic inflammation through the release of several pro-inflammatory mediators from the liver and/or visceral adipose tissues, such as increased reactive oxygen species, $\mathrm{C}$ reactive protein (CRP), IL-6, and other adipokines (26).

\section{MANAGEMENT OF PATIENTS WITH MODERATE TO SEVERE PSORIASIS AND METABOLIC COMORBIDITIES}

The association between psoriasis and cardio-metabolic disorders has important clinical consequences. Firstly, systemic treatments for psoriasis could negatively affect metabolic comorbidities, especially in case of continuous and prolonged use (Table 1). In particular, methotrexate should be prescribed with caution in the presence of obesity, diabetes, NAFLD, and heavy alcohol intake because of the increased risk of liver fibrosis (27). Transient elastography and FibroTest are effective noninvasive tools for monitoring hepatotoxicity in patients receiving methotrexate for psoriasis (28). Moreover, it should be considered that $\mathrm{CKD}$ of older patients could reduce renal clearance of methotrexate favoring toxicity (29). Cyclosporine can induce or worsen arterial hypertension, increase insulin resistance, and interfere with fatty acid metabolism inducing dyslipidemia and hyperuricemia (30). Then, the drug interaction between cyclosporine and statins, which are commonly used for hypercholesterolemia, could potentially induce rabdomiolysis (31). Consequently, cyclosporine should be used with caution in psoriatic patients with features of the metabolic syndrome. Moreover, the presence of an established CKD is a contraindication for cyclosporine use. Acitretin could also favor hypertriglyceridemia and/or hypercholesterolemia, although severe cases are rare (30). PUVA and narrowband UVB therapy are not expected to cause significant changes in metabolic parameters (32), but are impractical for many patients.

Generally, biological therapies do not negatively affect metabolic parameters as conventional treatments can do. However,

\section{Table 1 | Systemic treatments for psoriasis could negatively affect the components of metabolic syndrome}

\begin{tabular}{lccccc}
\hline & MTX & CsA & Acitretin & Anti-TNF- $\alpha$ & Ustekinumab \\
\hline Hyperlipidemia & No & Yes & Yes & No & No \\
Hypertension & No & Yes & No & No & No \\
Obesity & No & No & No & Yes & No \\
Diabetes & No & Yes & No & No & No \\
NAFLD & Yes & No & No & No & No \\
Decreased renal & Yes & Yes & No & No & No \\
function & & & & &
\end{tabular}

NAFLD, non-alcoholic fatty liver disease. 
clinically significant dyslipidemia has been occasionally reported in patients receiving TNF- $\alpha$ antagonists, but this is not a common issue in clinical practice (33). A body weight gain could occur in patients treated with TNF- $\alpha$ antagonists $(34,35)$. Weight changes are induced mainly by fat mass gain in patients with psoriasis receiving TNF- $\alpha$ antagonists (34). By contrast, ustekinumab is not associated to body weight increase in patients with chronic plaque psoriasis (36). The effects of anti-TNF therapy on insulin sensitivity is controversial $(37,38)$. The effects of TNF- $\alpha$ inhibitors on glycemic parameters and insulin resistance in patients with psoriasis have been addressed mostly in small studies by means of the Homeostasis Model Assessment (HOMA) and the Quantitative Insulin Sensitivity Check Index (QUICKI), two widely used non-invasive surrogate markers of insulin resistance and sensitivity, respectively. A randomized, double-blind study in 12 psoriatic patients at high risk of developing type 2 diabetes mellitus failed to see a significant effect of a 2 -week treatment with etanercept on insulin secretion and sensitivity (39). In contrast, a 24 -week study in nine patients with stable active plaque psoriasis treated with etanercept found a significant reduction in insulin plasma levels, with a significant improvement in insulin resistance as suggested by the decrease in the HOMA index (40). No significant changes in insulin sensitivity or in the levels of fasting blood glucose were seen in a study in 18 patients with psoriasis after 12 weeks of treatment with adalimumab (41). Solomon et al. found that patients with rheumatoid arthritis or psoriasis receiving TNF-inhibitors had lower risk of developing diabetes mellitus compared with other treatments (42).

Biologics targeting the IL-12/23 pathway have proven to be highly effective in psoriasis, but their safety on CDV risk is still a matter of controversy. Indeed, briakinumab was withdrawn from clinical development because of an imbalance of major CDV events in the treatment group compared to controls (43). Consequently, there have been similar concerns over the safety of ustekinumab, although data from international post-marketing registry does not show any risk (44). To date, the Food and Drug Administration has not issued changes to the prescribing information for ustekinumab related to CDV risk. It could be also considered that drugs used mainly in the treatment of arterial hypertension including beta-blockers and ACE-inhibitors may worsen psoriasis in some patients (45). Finally, patients with moderate to severe psoriasis are candidate to interventions aimed to reduce their CDV risk including hypo-caloric diet, regular physical activity, and smoking cessation. Low calorie diet inducing a moderate weight loss (i.e., $5-10 \%$ of body weight) increases the responsiveness of obese patients to any systemic treatments (4648). Smoking habit has been associated to onset and worsening of psoriasis, and smoking cessation can positively affect the disease course (49). Patients with psoriasis exhibit decreased levels of physical activity, possibly for both psychological and physiological reasons (50). Regular physical activity may lower the risk of incident psoriasis and have also a beneficial effect on the natural course of the disease influencing the severity as well as metabolic comorbidities (51).

From a clinical prospective, the understanding of the patients in the context of these comorbidities is very important to ensure that treatment is tailored to meet the individual patient needs.

\section{REFERENCES}

1. Tsoi LC, Spain SL, Knight J, Ellinghaus E, Stuart PE, Capon F, et al. Identification of 15 new psoriasis susceptibility loci highlights the role of innate immunity. Nat Genet (2012) 44:1341-8. doi:10.1038/ng.2467

2. Elder JT, Bruce AT, Gudjonsson JE, Johnston A, Stuart PE, Tejasvi T, et al. Molecular dissection of psoriasis: integrating genetics and biology. J Invest Dermatol (2010) 130:1213-26. doi:10.1038/jid.2009.319

3. Lowes MA, Bowcock AM, Krueger JG. Pathogenesis and therapy of psoriasis. Nature (2007) 445:866-73. doi:10.1038/nature05663

4. Nestle FO, Kaplan DH, Barker J. Psoriasis. N Engl J Med (2009) 361:496-509. doi:10.1056/NEJMra0804595

5. Gisondi P, Girolomoni G. Impact of TNF- $\alpha$ antagonists on the quality of life in selected skin diseases. G Ital Dermatol Venereol (2013) 148:243-8.

6. Gisondi P, Girolomoni G. Cardiometabolic comorbidities and the approach to patients with psoriasis. Actas Dermosifiliogr (2009) 100(Suppl 2):14-21. doi:10.1016/S0001-7310(09)73373-3

7. Armstrong AW, Harskamp CT, Armstrong EJ. The association between psoriasis and obesity: a systematic review and meta-analysis of observational studies. Nutr Diabetes (2012) 2:e54. doi:10.1038/nutd.2012.26

8. Setty AR, Curhan G, Choi HK. Obesity, waist circumference, weight change, and the risk of psoriasis in women: nurses' health study II. Arch Intern Med (2007) 13(167):1670-5. doi:10.1001/archinte.167.15.1670

9. Paller AS, Mercy K, Kwasny MJ, Choon SE, Cordoro KM, Girolomoni G, et al. Association of pediatric psoriasis severity with excess and central adiposity: an international cross-sectional study. JAMA Dermatol (2013) 149:166-76. doi:10.1001/jamadermatol.2013.1078

10. Soltani-Arabshahi R, Wong B, Feng B-J, Goldgar DE, Duffin KC, Krueger GG. Obesity in early adulthood as a risk factor for psoriatic arthritis. Arch Dermatol (2010) 146:721-6. doi:10.1001/archdermatol.2010.141

11. Davidovici BB, Sattar N, Prinz JC, Jörg PC, Puig L, Emery P, et al. Psoriasis and systemic inflammatory diseases: potential mechanistic links between skin disease and co-morbid conditions. J Invest Dermatol (2010) 130:1785-96. doi:10.1038/jid.2010.103

12. Coto-Segura P, Eiris-Salvado N, González-Lara L, Queiro-Silva R, MartinezCamblor P, Maldonado-Seral C, et al. Psoriasis, psoriatic arthritis and type 2 diabetes mellitus: a systematic review and meta-analysis. Br J Dermatol (2013) 169:783-93. doi:10.1111/bjd.12473

13. Buerger C, Richter B, Woth K, Salgo R, Malisiewicz B, Diehl S, et al. Interleukin-1 $\beta$ interferes with epidermal homeostasis through induction of insulin resistance: implications for psoriasis pathogenesis. J Invest Dermatol (2012) 132:2206-14. doi:10.1038/jid.2012.123

14. Gisondi P, Tessari G, Conti A, Piaserico S, Schianchi S, Peserico A, et al. Prevalence of metabolic syndrome in patients with psoriasis: a hospital-based casecontrol study. Br J Dermatol (2007) 157:68-73. doi:10.1111/j.1365-2133.2007. 07986.x

15. Armstrong AW, Harskamp CT, Armstrong EJ. Psoriasis and metabolic syndrome: a systematic review and meta-analysis of observational studies. J Am Acad Dermatol (2013) 68:654-62. doi:10.1016/j.jaad.2012.08.015

16. Targher G, Byrne CD. Clinical review: nonalcoholic fatty liver disease: a novel cardiometabolic risk factor for type 2 diabetes and its complications. J Clin Endocrinol Metab (2013) 98:483-95. doi:10.1210/jc.2012-3093

17. Gisondi P, Targher G, Zoppini G, Girolomoni G. Non-alcoholic fatty liver disease in patients with chronic plaque psoriasis. J Hepatol (2009) 51:758-64. doi:10.1016/j.jhep.2009.04.020

18. Van der Voort EAM, Koehler EM, Dowlatshahi EA, Hofman A, Stricker $\mathrm{BH}$, Janssen HLA, et al. Psoriasis is independently associated with nonalcoholic fatty liver disease in patients 55 years old or older: results from a population-based study. J Am Acad Dermatol (2014) 70:517-24. doi:10.1016/ j.jaad.2013.10.044

19. Targher G, Day CP, Bonora E. Risk of cardiovascular disease in patients with nonalcoholic fatty liver disease. N Engl J Med (2010) 363:1341-50. doi:10.1056/NEJMra0912063

20. Prey S, Paul C, Bronsard V, Puzenat E, Gourraud P-A, Aractingi S, et al. Cardiovascular risk factors in patients with plaque psoriasis: a systematic review of epidemiological studies. J Eur Acad Dermatol Venereol (2010) 24(Suppl 2):23-30. doi:10.1111/j.1468-3083.2009.03564.x

21. Samarasekera EJ, Neilson JM, Warren RB, Parnham J, Smith CH. Incidence of cardiovascular disease in individuals with psoriasis: a systematic review and meta-analysis. J Invest Dermatol (2013) 133:2340-6. doi:10.1038/jid.2013.149 
22. Armstrong EJ, Harskamp CT, Armstrong AW. Psoriasis and major adverse cardiovascular events: a systematic review and meta-analysis of observational studies. J Am Heart Assoc (2013) 2(2):e000062. doi:10.1161/JAHA.113.000062

23. Mehta NN, Yu Y, Pinnelas R, Krishnamoorthy P, Shin DB, Troxel AB, et al. Attributable risk estimate of severe psoriasis on major cardiovascular events. Am JMed (2011) 124:.e1-6. doi:10.1016/j.amjmed.2011.03.028

24. Wan J, Wang S, Haynes K, Denburg MR, Shin DB, Gelfand JM. Risk of moderate to advanced kidney disease in patients with psoriasis: population based cohort study. BMJ (2013) 347:f5961. doi:10.1136/bmj.f5961

25. Lu Y, Chen H, Nikamo P, Qi Low H, Helms C, Seielstad M, et al. Association of cardiovascular and metabolic disease genes with psoriasis. J Invest Dermatol (2013) 133:836-9. doi:10.1038/jid.2012.366

26. Gisondi P, Lora V, Bonauguri C, Russo A, Lippi G, Girolomoni G. Serum chemerin is increased in patients with chronic plaque psoriasis and normalizes following treatment with infliximab. Br J Dermatol (2013) 168:749-55. doi:10.1111/bjd.12118

27. Rosenberg P, Urwitz H, Johannesson A, Ros A-M, Lindholm J, Kinnman N, et al. Psoriasis patients with diabetes type 2 are at high risk of developing liver fibrosis during methotrexate treatment. J Hepatol (2007) 46:1111-8. doi:10.1016/j.jhep.2007.01.024

28. Lynch M, Higgins E, McCormick PA, Kirby B, Nolan N, Rogers S, et al. The use of transient elastography and fibrotest for monitoring hepatotoxicity in patients receiving methotrexate for psoriasis. JAMA Dermatol (2014) 150(8):856-62. doi:10.1001/jamadermatol.2013.9336

29. Balato N, Patruno C, Napolitano M, Patrí A, Ayala F, Scarpa R. Managing moderate-to-severe psoriasis in the elderly. Drugs Aging (2014) 31:233-8. doi:10.1007/s40266-014-0156-6

30. Gisondi P, Cazzaniga S, Chimenti S, Giannetti A, Maccarone M, Picardo M, et al. Metabolic abnormalities associated with initiation of systemic treatment for psoriasis: evidence from the Italian Psocare Registry. J Eur Acad Dermatol Venereol (2013) 27:e30-41. doi:10.1111/j.1468-3083.2012.04450.x

31. Neuvonen PJ, Niemi M, Backman JT. Drug interactions with lipid-lowering drugs: mechanisms and clinical relevance. Clin Pharmacol Ther (2006) 80:565-81. doi:10.1016/j.clpt.2006.09.003

32. Chappe SG, Roenigk HH, Miller AJ, Beeaff DE, Tyrpin L. The effect of photochemotherapy on the cardiovascular system. J Am Acad Dermatol (1981) 4:561-6. doi:10.1016/S0190-9622(81)70057-4

33. Lestre S, Diamantino F, Veloso L, Fidalgo A, Ferreira A. Effects of etanercept treatment on lipid profile in patients with moderate-to-severe chronic plaque psoriasis: a retrospective cohort study. Eur J Dermatol (2011) 21:916-20. doi:10.1684/ejd.2011.1548

34. Renzo LDI, Saraceno R, Schipani C, Rizzo M, Bianchi A, Noce A, et al. Prospective assessment of body weight and body composition changes in patients with psoriasis receiving anti-TNF- $\alpha$ treatment. Dermatol Ther (2011) 24:446-51. doi:10.1111/j.1529-8019.2011.01439.x

35. Gisondi P, Cotena C, Tessari G, Girolomoni G. Anti-tumour necrosis factoralpha therapy increases body weight in patients with chronic plaque psoriasis: a retrospective cohort study. J Eur Acad Dermatol Venereol (2008) 22:341-4. doi:10.1111/j.1468-3083.2007.02429.x

36. Gisondi P, Conti A, Galdo G, Piaserico S, De Simone C, Girolomoni G. Ustekinumab does not increase body mass index in patients with chronic plaque psoriasis: a prospective cohort study. Br J Dermatol (2013) 168:1124-7. doi:10.1111/bjd.12235

37. Stagakis I, Bertsias G, Karvounaris S, Kavousanaki M, Virla D, Raptopoulou A, et al. Anti-tumor necrosis factor therapy improves insulin resistance, beta cell function and insulin signaling in active rheumatoid arthritis patients with high insulin resistance. Arthritis Res Ther (2012) 14:141. doi:10.1186/ar3874

38. Campanati A, Ganzetti G, Di Sario A, Damiani A, Sandroni L, Rosa L, et al The effect of etanercept on hepatic fibrosis risk in patients with non-alcoholic fatty liver disease, metabolic syndrome, and psoriasis. J Gastroenterol (2013) 48:839-46. doi:10.1007/s00535-012-0678-9

39. Martínez-Abundis E, Reynoso-von Drateln C, Hernández-Salazar E, GonzálezOrtiz M. Effect of etanercept on insulin secretion and insulin sensitivity in a randomized trial with psoriatic patients at risk for developing type 2 diabetes mellitus. Arch Dermatol Res (2007) 299:461-5. doi:10.1007/s00403-007-0784-3
40. Marra M, Campanati A, Testa R, Sirolla C, Bonfigli AR, Franceschi C, et al. Effect of etanercept on insulin sensitivity in nine patients with psoriasis. Int $J$ Immunopathol Pharmacol (2007) 20:731-6.

41. Kofoed K, Clemmensen A, Mikkelsen UR, Simonsen L, Andersen O, Gniadecki R. Effects of anti-tumor necrosis factor therapy on body composition and insulin sensitivity in patients with psoriasis. Arch Dermatol (2012) 148:1089-91. doi:10.1001/archdermatol.2012.1753

42. Solomon DH, Massarotti E, Garg R, Liu J, Canning C, Schneeweiss S. Association between disease-modifying antirheumatic drugs and diabetes risk in patients with rheumatoid arthritis and psoriasis. JAMA (2011) 305:2525-31. doi:10.1001/jama.2011.878

43. Dommasch ED, Troxel AB, Gelfand JM. Major cardiovascular events associated with anti-IL 12/23 agents: a tale of two meta-analyses. J Am Acad Dermatol (2013) 68:863-5. doi:10.1016/j.jaad.2013.01.011

44. Papp KA, Strober B, Augustin M, Calabro S, Londhe A, Chevrier M. PSOLAR: design, utility, and preliminary results of a prospective, international, diseasebased registry of patients with psoriasis who are receiving, or are candidates for, conventional systemic treatments or biologic agents. J Drugs Dermatol (2012) 11:1210-7.

45. Abel EA, DiCicco LM, Orenberg EK, Fraki JE, Farber EM. Drugs in exacerbation of psoriasis. J Am Acad Dermatol (1986) 15:1007-22. doi:10.1016/S01909622(86)70265-X

46. Gisondi P, Del Giglio M, Di Francesco V, Zamboni M, Girolomoni G. Weight loss improves the response of obese patients with moderate-to-severe chronic plaque psoriasis to low-dose cyclosporine therapy: a randomized, controlled, investigator-blinded clinical trial. Am J Clin Nutr (2008) 88:1242-7.

47. Naldi L, Conti A, Cazzaniga S, Patrizi A, Pazzaglia M, Lanzoni A, et al. Diet and physical exercise in psoriasis: a randomized controlled trial. Br J Dermatol (2014) 170:634-42. doi:10.1111/bjd.12735

48. Jensen P, Zachariae C, Christensen R, Geiker NRW, Schaadt BK, Stender S, et al. Effect of weight loss on the severity of psoriasis: a randomized clinical study. JAMA Dermatol (2013) 149:795-801. doi:10.1001/jamadermatol.2013.722

49. Kinahan CE, Mazloom S, Fernandez AP. Impact of smoking on response to systemic treatment in patients with psoriasis: a retrospective case-control study. $\mathrm{Br}$ J Dermatol (2014). doi:10.1111/bjd.13359

50. Torres T, Alexandre JM, Mendonça D, Vasconcelos C, Silva BM, Selores M. Levels of physical activity in patients with severe psoriasis: a cross-sectional questionnaire study. Am J Clin Dermatol (2014) 15:129-35. doi:10.1007/s40257-0140061-0

51. Balato N, Megna M, Palmisano F, Patruno C, Napolitano M, Scalvenzi M, et al. Psoriasis and sport: a new ally? J Eur Acad Dermatol Venereol (2014). doi:10.1111/jdv.12607

Conflict of Interest Statement: Paolo Gisondi has been a consultant and/or speaker for Abbott, Janssen, Leo-Pharma, Lilly, Merck Sharp \& Dohme, Novartis, and Pfizer. Arturo Galvan has nothing to declare. Luca Idolazzi has nothing to declare. Giampiero Girolomoni has been a consultant, investigator, and/or speaker for AbbVie, Almirall, Amgen, Boeringher-Ingelheim, Celgene, Galderma, GlaxoSmithKline, Eli-Lilly, Janssen, Leo-Pharma, Merck Serono, Merck Sharp \& Dohme, Novartis, Otsuka, Pfizer, and Shiseido.

Received: 17 September 2014; accepted: 01 January 2015; published online: 21 January 2015.

Citation: Gisondi P, Galvan A, Idolazzi L and Girolomoni G (2015) Management of moderate to severe psoriasis in patients with metabolic comorbidities. Front. Med. 2:1. doi: 10.3389/fmed.2015.00001

This article was submitted to Dermatology, a section of the journal Frontiers in Medicine.

Copyright (C) 2015 Gisondi, Galvan, Idolazzi and Girolomoni. This is an open-access article distributed under the terms of the Creative Commons Attribution License (CC BY). The use, distribution or reproduction in other forums is permitted, provided the original author(s) or licensor are credited and that the original publication in this journal is cited, in accordance with accepted academic practice. No use, distribution or reproduction is permitted which does not comply with these terms. 